\title{
Robotized line-scan thermography combined with a new compressed sensing technology for investigating a painting on canvas artwork
}

\author{
Hai Zhang ${ }^{1}$, Mingli Zhang ${ }^{2}$, Stefano Sfarra ${ }^{3}$, Ahmad Osman ${ }^{4,5}$, Clemente Ibarra-Castanedo ${ }^{1}$ and \\ Xavier P.V. Maldague ${ }^{1}$ \\ ${ }^{1}$ Computer Vision and Systems Laboratory (CVSL), Department of Electrical and Computer \\ Engineering, Laval University, Quebec City, G1V 0A6, Canada. \\ ${ }^{2}$ Montreal Neurological Institute, McGill University, Montreal, H3A 2B1, Canada. \\ ${ }^{3}$ Department of Industrial and Information Engineering and Economics, University of L'Aquila, \\ L'Aquila, I-67100, Italy. \\ ${ }^{4}$ Department of Inspection of Components and Assemblies, Fraunhofer IZFP, 66123 Saarbruecken, \\ Germany. \\ 5University of Applied Sciences (htw saar), 66117 Saarbruecken, Germany
}

\begin{abstract}
This work presents a fast robotized line-scan thermography (LST) modality for the inspection of two paintings on canvas, which are the mock-ups of a famous oil painting, titled Portrait of the Painter's Mother (by James Abbott McNeill Whistler, 1871). Here, a new image restoration (IR) post-processing technique is proposed, which combines both external and internal information for the high-performance reconstruction of the LST data. In the IR technique, a probabilistic atlas is used to model the spatial distribution of gradients, which correspond to various anatomical structures in the LST data. This atlas is then employed to control the level of gradient regularization at each image location, within a weighted total variation regularization prior. IR also leverages the redundancy of nonlocal similar patches through a sparse representation model. Experiments show that IR method outperforms the current proposed approaches; this, for different sampling rates and noise levels. In addition, x-ray imaging was used for comparative purposes. It was concluded that the proposed LSTIR modality is an effective technique for artwork fast inspection, and it can additionally provide a higher image contrast if compared to the state-of-the-art post-processing modalities such as those commonly used in the classical pulsed thermography technique. To reach these conclusions, physical analyses were also conducted. Finally, this work appears useful to investigate the feasibility of a fast robotized modality for large-scale artwork in-line inspection.
\end{abstract}

